\title{
Ultrafast excited state deactivation and energy transfer in guanine-cytosine DNA double helices
}

\author{
François-Alexandre Miannay, Ákos Bányász, Thomas Gustavsson and Dimitra Markovitsi* \\ Laboratoire Francis Perrin, CEA/DSM/DRECAM/SPAM - CNRS URA 2453, CEA/Saclay, 91191 Gif-sur-Yvette, \\ France
}

RECEIVED DAT E (automatically inserted by publisher); dimitra.markovitsi@cea.fr

The stability of DNA components with respect to UV radiation is considered to be a prerequisite for the development of the genetic code. But it is also known that UV light absorbed by DNA bases may damage the double helix and lead to carcinogenic mutations. ${ }^{1}$ The interplay between stability and photodamage depends on the way that the energy of a UV photon is distributed among the electronic excited states of the double helix before it is eliminated as heat. Ultrafast dissipation of the excitation energy is indeed a common property of all the monomeric DNA building blocks: the major part of the excited state population of nucleosides and nucleotides in aqueous solution lives for less than one picosecond. ${ }^{2,3}$ When applied to double helices, composed exclusively of adenine-thymine base pairs (A-T duplexes, both homopolymeric and alternating), femtosecond spectroscopy reveals a different picture: organization of the bases within duplexes causes an overall lengthening of the excited state lifetimes. ${ }^{4-9}$ This is due to the emergence of new excited states, shared between at least two bases. The existence of delocalized excited states allows ultrafast energy transfer to occur ${ }^{5,8-10}$ by-passing the prerequisites of Förster transfer which are not fulfilled in the case of DNA bases. ${ }^{10}$

Here we present the first femtosecond study dealing with a double helix composed only of guanine-cytosine (G-C) pairs. We show that the excited state relaxation of poly $(\mathrm{dGdC})$. poly $(\mathrm{dGdC})$ is faster than that of the monomeric components 2'-deoxyguanosine monophosphate (dGMP) and 2'-deoxycytosine monophosphate (dCMP). This contrasts with the behavior of A-T duplexes determined under identical experimental conditions. ${ }^{4,5,7,8}$ Despite this difference in the excited state deactivation rate, the signature of ultrafast energy transfer is also present in data obtained for poly $(\mathrm{dGdC})$.poly $(\mathrm{dGdC})$. These two facts together could play an important role in the behavior of native DNA.

The excitation source of the time-resolved setup ${ }^{11}$ was a frequency tripled titane-sapphire laser $(267 \mathrm{~nm})$. The detection was based on fluorescence upconversion with an instrumental response function of $330 \mathrm{fs}$ ( $\mathrm{fwhm}$ ). The use of a flow-cell (optical path-length: $0.4 \mathrm{~mm}$ ) allowing the circulation of $25 \mathrm{ml}$ of solution (concentration: $10^{-3} \mathrm{M}$ per base) was crucial in order to minimize photodamage. The duplex poly (dGdC).poly (dGdC) (Amersham Biosciences; $c a$. 1000 base pairs) was dissolved in phosphate buffer. dGMP and dCMP (Sigma) were dissolved in ultrapure water (MilliQ) in order to avoid aggregation, promoted by the ionic strength.

Figure 1a shows the fluorescence decay of poly $(\mathrm{dGdC}) \cdot \operatorname{poly}(\mathrm{dGdC})$ at $330 \mathrm{~nm}$, together with those of dGMP and dCMP. At 1.4 ps, the duplex signal has lost $99 \%$ of its amplitude, whereas, at the same time, the amplitude of the signals observed for both nucleotides is about one order of magnitude higher. Upon increasing the emission wavelength, the duplex decays become slower (Figure 2) but they remain faster than those of the dGMP and dCMP.

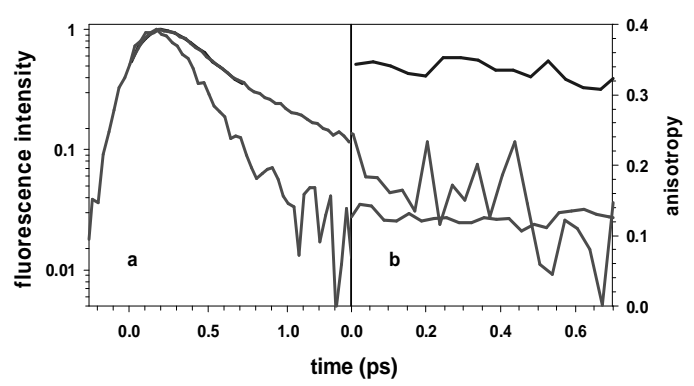

Figure 1. Fluorescence decays (a) and fluorescence anisotropy decays (b) of poly(dGdC).poly(dGdC) (red), dGMP (green) and dCMP (blue) recorded by the upconversion technique at $330 \mathrm{~nm}$. The zero time was defined at the half rise of the fluorescence signals.

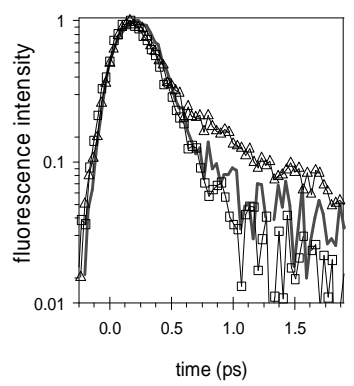

Figure 2. Fluorescence decays of poly $(\mathrm{dGdC}) \cdot \operatorname{poly}(\mathrm{dGdC})$ at $310 \mathrm{~nm}$ (squares), $330 \mathrm{~nm}$ (red lines) and $360 \mathrm{~nm}$ (triangles).

An acceleration of the excited state dynamics within G-C Watson-Crick (W-C) dimers is predicted by theoretical studies which point out the role of proton transfer. ${ }^{12-14}$ Quite recently, fluorescence upconversion measurements, carried out for guanosine and cytidine and their W-C pairs in chloroform, showed that base pairing reduces the excited state lifetimes. ${ }^{15}$

An important spectroscopic difference between G-C isolated pairs and the double strand resides on the electronic transitions involved in photon absorption. Indeed, the absorption spectrum of G-C pairs in chloroform is similar to the sum spectrum of the two monomeric chromophores ${ }^{15}$ whereas that of the G-C duplex is clearly different (Figure 3a). This double comparison reveals the magnitude of 
electronic interactions among bases in different W-C pairs. Calculations performed in the frame of the exciton theory taking into account conformational dynamics showed that dipolar coupling between $\pi \pi^{*}$ transitions of all twenty bases composing a $(\mathrm{dGdC})_{5} \cdot(\mathrm{dGdC})_{5}$ duplex induce delocalization of the Franck-Condon excited states over a small number of bases and alter the absorption spectrum. ${ }^{16}$ Consequently, laser excitation of poly $(\mathrm{dGdC})$.poly $(\mathrm{dGdC})$ at $267 \mathrm{~nm}$ populates a large number of excited states whose properties depend on the conformation of the double helix. Then, following intraband scattering (internal conversion among exciton states), emission may arise from excited states which are not directly excited resulting to energy transfer.

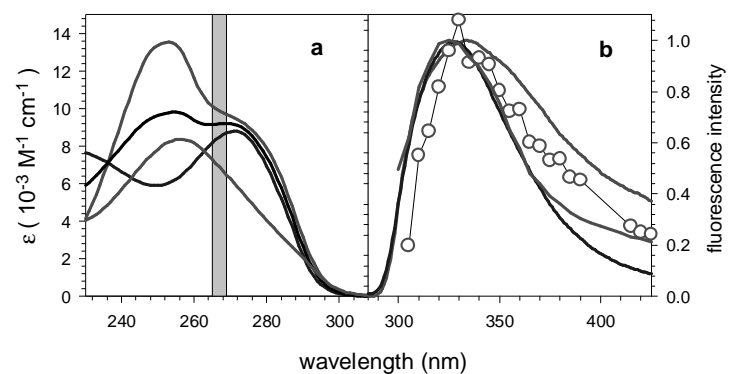

Figure 3. Steady-state absorption (a) and fluorescence (b) spectra of poly(dGdC).poly(dGdC) (red), dGMP (green), dCMP (blue) and an equimolar mixture of dGMP and dCMP (black). The grey bar indicates the laser excitation at $267 \mathrm{~nm}$. Red circles correspond to the timeresolved fluorescence spectrum of poly (dGdC).poly(dGdC). The steadystate fluorescence spectrum is taken from reference 17

The above processes were extensively studied for $(\mathrm{dA})_{\mathrm{n}} \cdot(\mathrm{dT})_{\mathrm{n}}$ duplexes for which we identified two experimental features relevant of intraband scattering: ${ }^{5,70}$ (i) the steady-state fluorescence spectrum of the duplex does not vary with the excitation wavelength, although it is composed of monomeric chromophores characterized by different absorption and fluorescence spectra; (ii) the fluorescence anisotropy of the duplexes decreases on the subpicosecond time scale, where molecular motions are inhibited. Such a behavior of fluorescence anisotropy was also found for alternating $(\mathrm{dAdT})_{\mathrm{n}} \cdot(\mathrm{dAdT})_{\mathrm{n}}$ duplexes. $^{8}$ Both of these experimental features are also observed for poly $(d G d C)$.poly (dGdC).

Figure $1 \mathrm{~b}$ shows that the fluorescence anisotropy of the duplex observed at $330 \mathrm{~nm}$ decays form ca. 0.25 to 0.1 within 0.7 ps. Since the data are convoluted by the instrumental response function; the true anisotropy may exhibit a much faster decrease. On the same time scale, the anisotropy of dGMP and dCMP remains constant, 0.13 and 0.35 , respectively. ${ }^{3}$ Regarding the steady state fluorescence spectrum of poly $(\mathrm{dGdC})$.poly $(\mathrm{dGdC})$, it was reported that it does not vary with the excitation wavelength. ${ }^{17}$ The spectrum recorded by fluorescence upconversion at the maximum of the fluorescence decays, peaks at $330 \pm 5 \mathrm{~nm}$ (Figure 3b) and resembles the steady-state spectrum reported by Huang and Georgiou. ${ }^{17}$ Since these two spectra have been obtained in different laboratories using different types of buffer, it is hard to affirm if the weak red shift of the time-resolved spectrum with respect to the steady-state one is significant. It is located in the same spectral region as that corresponding to $\pi \pi^{*}$ transitions of dGMP (peaking at $340 \mathrm{~nm}$ ) and dCMP (peaking at $325 \mathrm{~nm}$ ). This means that emission arises mainly from $\pi \pi^{*}$ states and not from states involving proton transfer whose energy is expected to be much lower. ${ }^{13}$ However, at longer times, partial contribution from charge transfer states, ${ }^{13,14,18}$ is quite possible.

The findings of the present experimental study constitute a challenge for further theoretical work, necessary to understand the contrast in the fluorescence decays between $\mathrm{G}-\mathrm{C}$ and A-T duplexes. The main question to answer is whether proton transfer within the W-C pairs is indeed the driving force for ultrafast excited state deactivation in G-C double helices. Or do other processes related to coupling among bases belonging to different $\mathrm{W}-\mathrm{C}$ pairs also play an important role? Do conical intersections between $\pi \pi^{*}$ and $\mathrm{n} \pi *$ states, evoked as one of the reasons for the short excited state lifetimes of nucleotides, ${ }^{19}$ shift to lower energies in G$\mathrm{C}$ helices further accelerating the ground state recovery? Finally, it is interesting to correlate the ultrafast fluorescence decays of poly(dGdC). poly (dGdC) with its low fluorescence quantum yield $\left(\mathrm{ca} \cdot 10^{-5}\right)$, similar to that of highly purified native DNA $\left(3 \times 10^{-5}\right) \cdot{ }^{17}$ Knowing that the fluorescence quantum yield of A-T duplexes is at least one order of magnitude higher, ${ }^{10,20}$ we can speculate that alternating GC sequences play the role of energy sinks or traps within native DNA.

Supporting Information Available: Experimental details, fits of fluorescence decays

\section{References}

(1) Melnikova, V. O.; Ananthaswamy, H. N. Mutat. Res. 2005, 571, 91106.

(2) Pecourt, J.-M. L.; Peon, J.; Kohler, B. J. Am. Chem. Soc. 2001, 123, 10370-10378; Peon, J.; Zewail, A. H. Chem. Phys. Lett. 2001, 348, 255262.

(3) Onidas, D.; Markovitsi, D.; Marguet, S.; Sharonov, A.; Gustavsson, T. J. Phys. Chem. B 2002, 106, 11367-11374.

(4) Markovitsi, D.; Sharonov, A.; Onidas, D.; Gustavsson, T. ChemPhysChem 2003, 3, 303-305.

(5) Markovitsi, D.; Onidas, D.; Gustavsson, T.; Talbot, F.; Lazzarotto, E. J. Am. Chem. Soc. 2005, 127, 17130-17131.

(6) Crespo-Hernández, C. E.; Cohen, B.; Kohler, B. Nature 2005, 436, 1141-1144; Markovitsi, D.; Talbot, F.; Gustavsson, T.; Onidas, D.; Lazzarotto, E.; Marguet, S. Nature 2006, 441, E7.

(7) Onidas, D.; Gustavsson, T.; Lazzarotto, E.; Markovitsi, D. J. Phys. Chem. B 2007, 111, 9644-9650.

(8) Onidas, D.; Gustavsson, T.; Lazzarotto, E.; Markovitsi, D. Phys. Chem. Chem. Phys. 2007, 9, 5143-5148.

(9) Buchvarov, I.; Wang, Q.; Raytchev, M.; Trifonov, A.; Fiebig, T. Proc. Natl. Acad. Sci. 2007, 104, 4794-4797.

(10) Markovitsi, D.; Gustavsson, T.; Talbot, F. Photochem. \& Photobiol. Sci. 2007, $717-724$.

(11) Gustavsson, T.; Sharonov, A.; Onidas, D.; Markovitsi, D. Chem. Phys. Lett. 2002, 356, 49-54.

(12) Sobolewski, A. L.; Domcke, W. Phys. Chem. Chem. Phys. 2004, 6, 2763-2771.

(13) Sobolewski, A. L.; Domcke, W.; Hättig, C. Proc. Natl. Acad. Sci. 2005, 102, 17903-17906.

(14) Groenof, G.; Schäfer, L. V.; Boggio-Pasqua, M.; Goette, M.; Grubmüller, H.; Robb, M. A. J. Am. Chem. Soc. 2007, 129, 6812-6819. (15) Schwalb, N.; Temps, F. J. Am. Chem. Soc. 2007, 129, 9272-9273.

(16) Emanuele, E.; Zakrzewska, K.; Markovitsi, D.; Lavery, R.; Millie, P. J. Phys. Chem. B 2005, 109, 16109-16118.

(17) Huang, C.-R.; Georghiou, S. Photochem. Photobiol. 1992, 56, 9599.

(18) Starikov, E. B. Modern Phys. Lett. B 2004, 18, 825-831; AboRiziq, A.; Grace, L.; Nir, E.; Kabelac, M.; Hobza, P.; de Vries, M. Proc. Natl. Acad. Sci. 2005, 102, 20-23.

(19) Hare, P. M.; Crespo-Hernández, C.; Kohler, B. Proc. Natl. Acad. Sci. 2007, 104, 435-440.

(20) Ge, G.; Georghiou, S. Photochem. Photobiol. 1991, 54, 301-305. 


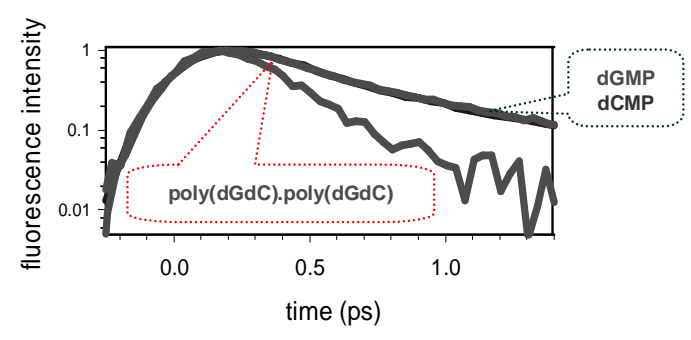

\section{ABSTRACT FOR WEB PUBLICATION}

The DNA double helix poly $(\mathrm{dGdC})$.poly $(\mathrm{dGdC})$ is studied by fluorescence upconversion spectroscopy with femtosecond resolution. It is shown that the excited state relaxation of the duplex is faster than that of the monomeric components dGMP and dCMP. This contrasts with the behavior of duplexes composed exclusively of adenine-thymine base pairs, for which an overall lengthening of the fluorescence lifetimes with respect to that of an equimolar mixture of dAMP and TMP was reported previously. Despite the difference in the excited state deactivation rate between the two types of duplexes, the signature of ultrafast energy transfer is present in both of them. It is attested by the decrease of fluorescence anisotropy decay of the duplexes on the sub-picosecond time scale, where molecular motions are inhibited, and is corroborated by the fact that their steady-state fluorescence spectra do not change with the excitation wavelength. Energy transfer involves excited states delocalized over at least two bases, whose existence is revealed by the UV absorption spectrum of the duplex, clearly different from that of an equimolar spectrum of dGMP and dCMP. 


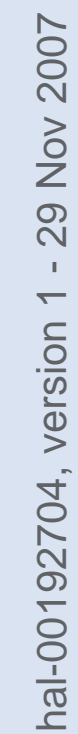

